\title{
Traumatic Spinal Cord Injuries: An Evidence-based Practice Approach Kumar $\mathbf{N}^{*}$
}

Consultant in Spinal Injuries and Rehabilitation Medicine, Robert Jones and Agnes Hunt Orthopaedic Hospital, Oswestry, UK

\begin{abstract}
This article deals with the main issues of management of traumatic spinal cord injury discussing - physiological instability, prognostic determinants of recovery, natural history, care in supine position, mobilization, canal encroachment, cord compression, and indications for surgery.
\end{abstract}

A review of relevant literature has been done including the STASCIS study to try and explore whether early or late surgery for traumatic spinal cord injury is better than conservative management.

It reflects the ethos of Active Physiological Conservative Management for these patients at RJ\&AH Orthopaedic Hospital.

Considering the lack of credible evidence demonstrating superiority of outcome with surgery compared to early active management of the traumatic spinal injury, patients should be encouraged to make an informed choice.

Keywords: Trauma; Spinal cord injury; Surgery; Conservative management; Evidence-based practice

\section{Introduction}

The Management of the Traumatic spinal injury has remained controversial since the times of Charles Bell and Astley Cooper. Traumatic spinal injuries in 1920's and 1930's were non-operatively managed. Methods of reduction included hyperextension in the prone position on slings, frames or hammock, as described by Davis and Rogers or hanging (Bohler). Watson-Jones used the two-table method in 1931 and 1934. Dunlop and Parker hyper extended the broken spine in supine position.

Magnus renounced methods of forceful reduction and accepted the spinal deformity. He advocated allowing the fractured spine to consolidate by placing the patient flat in bed in supine position with prolonged recumbency for 3 to 6 months or longer. Such prolonged immobilisation was carried out with often poor attention and poor management of the associated multisystem physiological impairment and malfunction. These methods of management have been strongly condemned (Guttmann, Watson-Jones, Holdsworth and Hardy) as utterly contrary to the principles of rehabilitation of spinal cord injured patients.

In 1944, Guttmann introduced and developed the method of graduated reduction of fractures and fracture dislocations of the injured spine and immobilisation on pillow packs while providing simultaneous detailed attention to the multisystem malfunction together with all medical and non-medical effects of paralysis. $\mathrm{He}$ demonstrated that almost all of the complications that were believed to be inevitable following a SCI were indeed preventable. He asserted that complications following SCI are attributable to poor management of the patient rather than the neurological impairment or the patient being treated with bed rest. Interestingly although anatomical alignment was rarely achieved, Guttmann demonstrated that with simultaneous attention to all medical and non-medical effects of the SCI a significant number of patients recovered motor and sensory functions to ambulate and the majority were pain free following conservative management.

Based on such evidence the Active Physiological Conservative Management (APCM) of the spinal injury and its effects was described and popularised by Wagih El Masri pupil of Guttmann. El Masri, et al. [1] demonstrated that with expert, early, simultaneous APCM of the injured spine, spinal cord and all the medical and non-medical effects over $70 \%$ of patients with complete motor paralysis but with sparing of pin prick sensation presenting in the first 72 hours of injury recover motor power to ambulate without surgical, pharmacological, cellular or biological intervention. Those presenting within 72 hours of injury with motor sparing, however minimal the sparing is, have an even better chance to walk, also without any intervention. El Masri et al., also demonstrated that the reduction of the period of treatment in recumbence from 12 weeks to between 4-6 weeks was safe both in the short and long term. They also demonstrated that with APCM the impact on the patient and family members can be minimised in both the short and long term. Patients who do not recover ambulation can with APCM and ongoing expert monitoring, care and support lead dignified, healthy, fulfilling, productive and often competitive lives [1-3].

\section{Active Physiological Conservative Management (APCM)}

Active Physiological Conservative Management, from the early hours of injury requires simultaneous scrupulous care of: the injured spine, the multisystem neurogenic effects of the spinal cord injury on the respiratory, cardiovascular, urinary, gastrointestinal, dermatological, sexual and reproductive functions, the management of the associated psychological effects of paralysis, the physical rehabilitation and the modification of the environment. By definition APCM requires between 4-6 weeks of treatment in recumbence. This is in order to rest injured tissue, prevent significant postural hypotension or significant reduction of vital capacity during the stage of spinal shock, minimise the risk of ischial and sacral pressure sores during the vulnerable period of poor skin perfusion due to spinal shock, facilitate intermittent catheterisation, facilitate bowel care and facilitate nursing care during the first few weeks of paralysis. It also allows for some recovery of the sympathetic nervous system reflexes which are paramount for the active cooperation of the patient with the demands of physical rehabilitation.

The ultimate goals of management are to ensure maximum neurological recovery and independence, a pain free and flexible spine, safe functioning of the various systems of the body with minimal

${ }^{*}$ Corresponding author: Naveen Kumar, Consultant in Spinal Injuries and Rehabilitation Medicine, Robert Jones and Agnes Hunt Orthopaedic Hospital, Oswestry, UK, Tel: +44 1691 404000; E-mail: Naveen.Kumar@rjah.nhs.uk

Received February 25, 2016; Accepted March 08, 2016; Published March 10, 2016

Citation: Kumar N (2016) Traumatic Spinal Cord Injuries: An Evidence-based Practice Approach. J Spine S7: 006.doi:10.4172/2165-7939.S7-006

Copyright: @ 2016 Kumar N. This is an open-access article distributed under the terms of the Creative Commons Attribution License, which permits unrestricted use, distribution, and reproduction in any medium, provided the original author and source are credited. 
inconvenience to patients and the prevention/minimisation of complications in both the short and the long term. It is equally important to enable patients to regain assertiveness, take control of their own lives, re-engage in activities of their choice and whenever possible compete in some spheres of life. The benefits to both the patient and the society, the education of patients and on-going support to maintain health and independence following discharge cannot be overemphasised.

\section{Physiological Instability}

It is understandable that the majority of those who manage SCI patients in the acute phase have concerns about the biomechanical instability at the fracture site and displacement or further displacement causing damage or further damage to neural tissues. It is also understandable that the "belief" that canal encroachment and cord compression may prevent neurological recovery or cause neurological deterioration is indeed logical.

The facts however are that in recumbence and with some careful handling of the patient neurological deterioration are very rare in the most biomechanically unstable injuries. Similarly vertebral misalignment, canal encroachment and cord compression do not prevent neurological recovery and are rarely, individually or in combination the cause of neurological deterioration when patients are adequately managed with APCM. These concerns will be dealt with in the next paragraphs.

What is often overlooked is that the injured cord is physiologically unstable because of the cellular and cell membrane disturbances, loss of auto regulatory functions and disruption of blood brain barrier [4] The physiologically unstable injured spinal cord cannot protect itself from non-mechanical complications outside the spinal canal such as hypoxia, hypotension, hypertension, sepsis and hypothermia. These complications hardly cause paralysis in the neurologically intact patients. In a patient with spinal cord injury and a physiologically unstable spine these complications can be at least as damaging to the injured neural tissues as the potential mechanical damage caused by the mismanagement of the Biomechanical Instability of the injured column.

\section{Biomechanical Instability}

The diagnosis of Biomechanical Instability is usually based on radiological investigations at the time of the presentation of the patient. Clinically and radiologically most vertebral fractures heal within 6- 12 weeks from injury when biomechanical stability is restored. Ligamentous injuries, however, can take longer to heal. Biomechanical Instability is therefore time related. The aim of the management of the Biomechanical Instability (surgically or with APCM) is therefore the "containment" of the instability until tissue healing occurs and stability restored. Biomechanical Instability is safely contained in recumbence for 4-6 weeks followed by bracing for a further six weeks during mobilisation and active rehabilitation. With APCM the great majority of injuries become biomechanically stable and pain free.

There is no evidence to suggest that surgical stabilisation enhances the speed of healing of injured tissue or achieves stability earlier than with APCM.

Admittedly the degree of kyphotic deformity is lower following surgical stabilisation than following APCM. The greatest majority of these residual kyphotic deformities in patients treated with APCM are however painless. The discrepancy between deformity and pain has been known for some time [5].
It must be appreciated that for wheelchair dependant patients a painless kyphotic deformity is likely to enhance independence and is certainly, much more preferable to a stiff straight neck or back following surgery.

\section{Prognostic Determinants of Recovery}

The neurological findings during the first 48-72 hours from injury are essential in predicting neurological recovery. Over $80 \%$ of tetraparetic patients who present within the first 72 hours from injury with any distal movement, however little and patchy (Frankel C) and over $70 \%$ of patients who present within the first 72 hours from injury with no motor power but with preservation of pin prick sensation down to S3 (Frankel B) will recover to walk again if they have not been harmed by the treatment [6-8]. Patients with complete cord injury (Frankel A) and pin prick sensation in the zone of partial preservation will recover significantly and regain useful motor power in these myotomes [7-9]. A neurological level higher than the bony level of fracture is another good prognostic indicator of zonal recovery $[9,10]$.

\section{Canal Encroachment}

Some of the first case reports to suggest that traumatic canal encroachment as demonstrated by computerised tomography does not correlate with the degree of neurological impairment, does not prevent neurological recovery and does not result in neurological deterioration were published by El Masri et al., in 1992 [11,12]. The same conclusions were made by reviewing the outcome of conservative treatment of 50 consecutive patients with between $10 \%$ to $90 \%$ canal encroachment in Frankel C, D and E groups; patients in Frankel C and D group recovered ambulation and none of the patients deteriorated neurologically or otherwise [13] Other groups have since published similar findings [14-16].

\section{Cord Compression}

In humans traumatic cord compression does not appear to prevent neurological recovery in patients with traumatic incomplete cord injuries 13, 6, and 14. Since the installation of the MRI scanner in our institution we have been monitoring (both prospectively and retrospectively) the neurological progress of conservatively managed patients with cord compression. The preliminary results indicate that the same clinical prognostic indicators of recovery apply whether the cord is compressed or not.

Furthermore surgical decompression of the injured spinal cord within 48 hours of injury results in a well-documented increase in the intrathecal pressure below the level of the decompression which could further compromise spinal cord perfusion causing overt neurological deterioration or silent neurological deterioration when expected recovery is not achieved. There is enough evidence in the literature to suggest that surgical decompression does not seem to be beneficial in either the laboratory animal or in humans when the severity of the initial impact is beyond a certain magnitude, in which case recovery will not occur [17-19]. This may explain why the great majority of patients with complete spinal cord injuries do not recover following decompression. There is no credible evidence to suggest that recovery of neural functions following decompression is due to the decompression.

To date there is no evidence to suggest that surgical decompression achieves better or earlier neurological recovery than APCM in humans with complete and /or incomplete cord or cauda equina injury

Some however advocate early surgical decompression within four hours of injury. This is based on experimental findings in rodents, cats and dogs with 20-60 million years of evolution behind humans. 
Translation from the laboratory animal to the clinical situation requires caution [20]. The logistics of adequate reliable clinical and electrophysiological assessment of neurological loss and sparing and of achieving surgical decompression within the first four hours of injury remain unfortunately unresolved.

\section{Natural History}

Fewer than $10 \%$ of patients initially with clinically complete spinal cord injuries (Frankel grade A, "FA") improve to make a significant recovery to ambulate with APCM [21]. Many more however, recover cord functions in one to four myotomal distributions below the level of the injury or improve to FB and FC18. Although since the 1980s anterior surgical decompression and arthrodesis have become increasingly established practice, based on suggestions that surgery resulted in motor zonal improvement; to date there is no evidence that surgery provides added value.

A series of 53 consecutive patients with complete traumatic tetraplegia, admitted to one centre within two days of injury, demonstrated that similar results can be achieved without surgical decompression or arthrodesis [8].

Patients with incomplete cord injuries make significant neurological recovery irrespective of the degree of canal stenosis, canal encroachment, misalignment or cord compression $[5,7,15,22]$ provided both the biomechanical Instability of the spinal column and the Physiological Instability of the spinal cord are well contained by APCM. Although almost every patient in our institution is given informed choice between conservative and surgical management the majority of patients with SCI chose APCM.

\section{Mobilisation}

Early mobilisation is advantageous to neurologically intact patients with stable fractures or following surgical stabilisation of unstable fractures. These patients can ambulate and be discharged to their own homes soon after surgery.

Patients with paralysis, general physiological impairment and multisystem malfunction do not benefit from early mobilisation, which may indeed be deleterious in more than one way to the patient. Individuals with spinal cord injury exhibit reduced lung volumes and flow rates as a result of respiratory muscle weakness. These features have been investigated in relation to the combined effects of injury level and posture. Values of forced vital capacity and forced expiratory volume in 1s (FEV (1)) were repeatedly and consistently shown to be larger in recumbence compared with the seated posture [23-26].

Early mobilisation of patients with spinal neural tissue injury is associated with a reduction of vital capacity [23-28] and a potential drop of oxygen saturation. Early mobilisation during the stage of spinal shock usually causes marked postural hypotension. The tetraplegic and high paraplegic patient's ability to cough is markedly impaired. It is more difficult to get rid of bronchial secretions with assisted coughing against gravity than when patients are in recumbence.

Individually or in combination these respiratory and vascular pathophysiological mechanisms can potentially cause further impairment of cord functions. It is more difficult to carry out intermittent catheterisation, bowel evacuation or manage episodes of urinary or bowel incontinence in a wheelchair than in recumbence. During the stage of spinal shock, the skin cord perfusion is markedly diminished and the skin over bony prominences is at its peak in vulnerability. While in recumbence the weight of the patients is spread across the body and all the bony prominences; in the wheelchair most of the weight of the patient is usually concentrated over the skin of the ischial tuberosities and the sacrum, increasing the risk of pressure sores over these bony prominences.

Furthermore there are no comparative studies to demonstrate added value of early mobilisation of patients with SCI following Surgical and Non-Surgical Management in: reducing the number of patients' bed days in recumbence throughout the first admission, the time to completion of equivalent end points of rehabilitation, the period of total hospitalisation, the incidence of ischial and sacral pressure sores, respiratory infections, urinay infections and other complications, the incidence of chronic back pain, the frequency of readmission following first discharge or the total period of hospitalisation during readmission for the treatment of various complications $[4,5,11]$.

\section{Indications for Surgery}

Certain groups of patients are likely to benefit from surgery and should be encouraged to consider the option. Neurologically intact patients with Physiologically Stable neural tissue but Biomechanically Unstable Spines are less at risk from neuro-physiological deterioration than the neurologically impaired. The neurologically intact patient does not require intensive prolonged treatment and rehabilitation, and can be discharged a few days following surgery.

The neurologically impaired and neurologically intact patients with uncontrolled epilepsy, the mentally challenged and patients who are unable to comply with bed rest on the balance of probability are safer with surgical stabilisation than with non-surgical stabilisation.

Patients with Biomechanical Instability from pure ligamentous injuries without bony injury are at risk of developing late painful deformities and indeed may benefit from surgery.

We suggest that it would be better for surgery to be postponed until the blood brain barrier is restored which is likely to take about three weeks following the injury. Patients who exhibit signs of neurological deterioration with evidence of further neurological compression of neural tissues on MRI may benefit from surgical decompression

Unfortunately surgery has become the preferred method of management, also known as the "Standard of Care", of traumatic spinal injuries. Currently, over $80 \%$ of patients with traumatic spinal cord injury (TSCI) are surgically decompressed and stabilised without the rigours of adequate research methodology or demonstration of superiority of neurological and/or other outcomes over APCM. This can be contrasted with practice in our orthopaedic institution (with five dedicated Spinal Surgeons) where the great majority of patients with TSCI are treated conservatively. A recent audit revealed that only $2.8 \%$ of patients who are transferred with virgin spines to our Institution are surgically managed [29].

\section{Surgical Timing in the Acute Spinal Cord Injury Study (STASCIS)}

The debate over the effects of the timing of surgical spinal decompression after traumatic spinal cord injury (TSCI) has remained unresolved for over a century. The Surgical Timing in the Acute Spinal Cord Injury Study (STASCIS) by Fehlings et al., reported that surgical decompression prior to 24 hours is safe and improved neurologic outcome more than late decompression (after $24 \mathrm{hrs)} \mathrm{[30].}$ The improvement was defined as recovery of at least 2 AIS grades at 6 months follow-up [30]. This drew criticism for: Poor sample size calculation, lack of significant difference for one AIS group, technically 
questionable analytical approach and Odds ratio in the early surgery group not meeting statistical significance [31]. Van Middendorp performed a systematic review and quality-adjusted meta-analysis of studies (1966 to August 2012) evaluating the effects of the timing of spinal surgery after TSCI [32]. He concluded that the published evidence lacks robustness as a result of different sources of heterogeneity within and between original studies.

Nevertheless assuming early decompression has better neurological outcome than late decompression; a critical in depth comparison of Frankel's outcome of APCM with Fehlings' outcome of early decompression will readily demonstrate APCM's superiority of neurological outcomes when adjustment has been made for the differences in the definitions between the various grades in the ASIA Impairment Scale (AIS) and the Frankel Scale.

\section{Conclusions}

To date there is no evidence to suggest that the surgical decompression or stabilisation of the neurologically impaired spinal cord injured patient is advantageous.

Until credible evidence is available to demonstrate superiority of outcome with early surgery compared to early active treatment of the injured spine, patients should be encouraged to manage an informed choice.

The ultimate goals of management should be to ensure maximum neurological recovery, independence, a pain free flexible spine, safe functioning of the various systems of the body with minimal or no inconvenience to patients and prevention or minimisation of complications.

\section{Acknowledgements}

The author would like to thank Prof. El Masri, Mr Osman and Mr Chowdhury, for their guidance and support without which the publication of this review article would not have been possible.

\section{References}

1. El Masri W (2010) Management of traumatic spinal cord injuries: current standard of care revisited. ACNR 10: 37-40.

2. Katoh S, El Masry WS (1995) Motor recovery of patients presenting with motor paralysis and sensory sparing following cervical spinal cord injuries. Paraplegia 33: 506-509.

3. Katoh S, el Masry WS (1994) Neurological recovery after conservative treatment of cervical cord injuries. J Bone Joint Surg Br 76: 225-228.

4. El Masry WS (1993) Physiological instability of the spinal cord following injury. Paraplegia 31: 273-275.

5. El Masry WS (2006) Traumatic spinal cord injury: the relationship between pathology and clinical implications. Trauma 8: 29-46.

6. Folman Y, el Masri W (1989) Spinal cord injury: prognostic indicators. Injury 20: 92-93.

7. Katoh S, El Masry WS, Jaffray D, McCall IW, Eisenstein SM, et al. (1996) Neurologic outcome in conservatively treated patients with incomplete closed traumatic cervical spinal cord injuries. Spine 21: 2346-2351.

8. Katoh S, el Masry WS (1994) Neurological recovery after conservative treatment of cervical cord injuries. J Bone Joint Surg Br 76: 225-228.

9. Katoh S, el Masry WS, Jaffray D, McCall IW, Eisenstein SM, et al. (1996) Neurologic outcome in conservatively treated patients with incomplete closed traumatic cervical spinal cord injuries. Spine (Phila Pa 1976) 21: 2345-2351.

10. El Masri WS, Kumar N (2011) Traumatic spinal cord injuries. Lancet 377: 972 974.

11. El Masri WS, Jaffray D (1992) Recent developments in the management of injuries of the cervical spine. In: Vinken PJ, Bruyn GW, Klawans HL, Frankel HL (eds.) Spinal cord trauma. Elsevier Science, Handbook of Clinical Neurology.

12. El Masri WS, Meerkotter DV (1992) Early decompression of the spinal cord following injury: arguments for and against. In: Illis LS (eds.) Spinal cord dysfunction Vol II: intervention and treatment. Oxford University Press, New York.
13. El Masri WS, Katoh S, Khan A (1995) Reflections on the neurologica significance of bony canal encroachment following traumatic injury of the spine in patients with Frankel C, D and E presentation. J Neurotrauma 10: 70.

14. Limb D, Shaw DL, Dickson RA (1995) Neurological injury in thoracolumbar burst fractures. J Bone Joint Surg Br 77: 774-777.

15. Rosenberg N, Lenger R, Weisz I, Stein H (1996) Neurological deficit in a consecutive series of vertebral fractures patients with bony fragments within the spinal canal. Spinal Cord 35: 92-95.

16. Boerger TO, Limb D, Dickson RA (2000) Does 'canal clearance' affect neurological outcome after thoracolumbar burst fractures? J Bone Joint Surg $\mathrm{Br}$ 82: 629-635.

17. Dolan EJ, Tator CH, Endrenyi L (1980) The value of decompression for acute experimental spinal cord compression injury. J Neurosurg 53: 749-755.

18. Guha A, Tator CH, Endrenyi L, Piper I (1987) Decompression of the spinal cord improves recovery after acute experimental spinal cord compression injury. Paraplegia 25: 324-339.

19. Tator CH, Duncan EG, Edmonds VE, Lapczak LI, Andrews DF (1987) Comparison of surgical and conservative management in 208 patients with acute spinal cord injury. Can J Neurol Sci 14: 60-69.

20. Courtine G, Bunge MB, Fawcett JW, Grossman RG, Kaas JH, et al. (2007) Can experiments in nonhuman primates expedite the translation of treatments for spinal cord injury in humans?. Nat Med 13: 561-566.

21. Frankel HL, Hancock DO, Hyslop G, Melzack J, Michaelis LS, et al. (1969) The value of postural reduction in initial management of closed injuries of the spine with paraplegia and tetraplegia. Paraplegia 7: 179-192.

22. Ibrahim A, Li Y, Li D, Raisman G, El Masry WS (2006) Olfactory ensheathing cells: ripples of an incoming tide? Lancet Neurol 5: 453-457.

23. Morgan MDL, Silver JR, Williams SJ (1986) The respiratory system of the spinal cord patient. In: Bloch RF, Basbaum M (eds) Management of spinal cord injury. Williams and Wilkins, Baltimore.

24. Cameron GS, Scott JW, Jousse AT, Botterell EH (1955) Diaphragmatic respiration in the Quadriplegic patient and the effect of position on his vital capacity. Ann Surg 141: 451-456.

25. Baydur A, Adkins RH, Milic-Emili J (2001) Lung mechanics in individuals with spinal cord injury: effects of injury level and posture. J Appl Physiol (1985) 90 405-411.

26. Alvisi V, Marangoni E, Zannoli S, Uneddu M, Uggento R, et al. (2012) Pulmonary function and expiratory flow limitation in acute cervical spinal cord injury. Arch Phys Med Rehabil 93: 1950-1956.

27. Carvell JE, Grundy DJ (1994) Complications of spinal surgery in acute spina cord injury. Paraplegia 32: 389-395.

28. Aung TS, el Masry WS (1997) Audit of a British Centre for spinal injury. Spinal Cord 35: 147-150.

29. Patil S, Myint M, Kumar N (2001) Indications and outcomes of surgical management of acute traumatic spinal cord injury (TSCI). Eur Spine J 2: 7-27.

30. Fehlings MG, Vaccaro A, Wilson JR, Singh A, W Cadotte D, et al. (2012) Early versus delayed decompression for traumatic cervical spinal cord injury: results of the Surgical Timing in Acute Spinal Cord Injury Study (STASCIS). PLoS One 7: e32037.

31. Fehlings MG, Vaccaro A, Wilson JR, Singh A, W Cadotte D, et al. (2012) Early versus delayed decompression for traumatic cervical spinal cord injury: results of the Surgical Timing in Acute Spinal Cord Injury Study (STASCIS). PLoS One7: e32037.

32. van Middendorp JJ, Hosman AJ, Doi SA (2013) The effects of the timing of spinal surgery after traumatic spinal cord injury: a systematic review and metaanalysis. J Neurotrauma 30: 1781-1794.

This article was originally published in a special issue, Spinal Cord Injury Rehabilitation handled by Editor(s). Dr. Alessandro Landi, University of Rome, Italy 